\title{
Propiedades de lechos fijos durante la deshidratación convectiva de cerezas, guindas y rosa mosqueta. Cambios de volumen y porosidad
}

\author{
Propriedades de leitos fixos durante a secagem por convecção de cerejas doces e \\ ácidas e frutas de rosa mosqueta. Mudanças de volume e porosidade
}

\author{
Carlos Alberto MÁRQUEZ ${ }^{1}$, Antonio DE MICHELIS ${ }^{2 *}$
}

\section{Resumen}

En este trabajo, se determinaron experimentalmente y modelaron los cambios de volumen y porosidad de lecho fijo durante la deshidratación de cerezas, guindas y frutos de la rosa mosqueta. Se utilizaron un equipo de secado experimental y frutas frescas. La altura de lecho variaba entre 0,04 y $0,12 \mathrm{~m}$, la temperatura del aire entre 50 y $70{ }^{\circ} \mathrm{C}$, su velocidad entre 1 y $4 \mathrm{~m} / \mathrm{s}$, y su humedad relativa fue de 5 y $50 \%$. Al inicio y durante la deshidratación se medía la altura del lecho como promedio de 8 escalas graduadas colocadas simétricamente en la cámara de secado y el peso de las muestras correspondientes. Con los valores obtenidos se calculaba el volumen de lecho y su porosidad en función del contenido de agua de la muestra. Mediante regresión, se obtuvieron correlaciones para estimar el volumen y la porosidad de lecho. Los errores porcentuales obtenidos fueron: para cambios en el volumen de lecho, entre $-16,4$ y 23,3 para cerezas y guindas, y entre $-4,9$ y 4,4 para frutos de la rosa mosqueta; y para los cambios de porosidad, entre $-15,2$ y 21,1 para cerezas y guindas, y entre $-2,6$ y 6,1 para frutos de la rosa mosqueta.
\end{abstract}

Palabras-clave: secado; lechos; cambios de volumen y porosidad; modelado.

\section{Resumo}

Neste trabalho foram determinadas e modeladas as mudanças de volume e porosidade de cerejas doces e ácidas, bem como de frutas de rosa mosqueta em leitos fixos durante a secagem. Foi usado um equipamento de secagem experimental e frutas frescas. A espessura dos leitos variou entre 0,04 e $0,12 \mathrm{~m}$, a temperatura do ar entre 50 e $70{ }^{\circ} \mathrm{C}$, a velocidade entre 1 e $4 \mathrm{~m} / \mathrm{s}$ e a umidade relativa de 5 a $50 \%$. Para o começo e durante a secagem foram medidos a altura do leito por meio de 8 escalas graduadas colocadas simetricamente na câmara de secagem e o peso das amostras correspondentes. Com os valores obtidos foi calculado o volume de leito e a porosidade em função do conteúdo de água da amostra. Por meio de correlações de regressão foram obtidos o volume e a porosidade de leito. Os erros porcentuais obtidos foram: para mudanças no volume de leito entre -16,4 e 23,3 para cerejas doces e ácidas, -4,9 e 4,4 para frutas de rosa mosqueta; e para as mudanças de porosidade entre -15,2 e 21,1 para cerejas doces e ácidas, e -2,6 e 6,1 para frutas de rosa mosqueta.

Palavras-chave: secagem; leitos; mudanças de volume e porosidade; modelagem.

\section{Introducción}

La deshidratación constituye un método fundamental en el procesamiento, almacenamiento y conservación de muchos productos alimenticios naturales como frutas, vegetales y granos. En general este proceso implica, a menos que el material haya sido sometido a pre tratamientos que destruyan la estructura celular, la deshidratación de tejidos celulares "vivos". A medida que avanza la deshidratación, las células evolucionan desde una estructura en equilibrio altamente organizada hasta un conjunto desordenado de los constituyentes fundamentales, debido a la deformación del material. Así, los mecanismos y propiedades de transporte resultan fuertemente dependientes de la naturaleza del material, su contenido de humedad y su temperatura, y consecuentemente, de su cambio de dimensiones (TELIS-ROMERO; SOBRAL, 2003).

Tanto el modelado como el diseño y la optimización de secaderos para alimentos de relativamente alto contenido de hu- medad requieren la formulación de ecuaciones que representen el proceso de deshidratación en forma realista, particularmente los balances de materia y energía. Para resolver los mencionados balances se deben considerar los cambios de volumen y de área superficial de las partículas individuales y la velocidad con que ocurren estos cambios en términos de las variables de operación del sistema. Dichos cambios también afectan, principalmente los del volumen y la porosidad, el comportamiento de lechos más o menos profundos de las mismas partículas. Los fenómenos indicados influyen considerablemente en las propiedades de transporte internas y externas de las partículas, obviamente también afectan los volúmenes de control para la resolución de los balances que serán variables, y tienen influencia en otros parámetros del diseño de equipos como, por ejemplo, la potencia de los ventiladores que impulsan el aire de secado, ya que esta depende, entre otros, de la altura y porosidad de los lechos de

Recebido para publicação em 13/9/2006

Aceito para publicação em 19/2/2008 (001849)

${ }^{1}$ Asentamiento Universitario Villa Regina, Facultad de Ingeniería, Universidad Nacional del Comahue, 25 de Mayo y Reconquista, 8436, Villa Regina, Río Negro, Argentina,

E-mail:cam_ing2003@yahoo.com.ar

2 CONICET. INTA AER El Bolsón. Mármol, 1950 (8430), El Bolsón, Río Negro, Argentina, E-mail: ademichelis@bariloche.inta.gov.ar

${ }^{*}$ A quem a correspondência deve ser enviada 
partículas (TELIS-ROMERO; SOBRAL, 2003). Asimismo, en la bibliografía se encuentran muy pocos datos de propiedades de lechos de partículas de frutas y hortalizas que deshidratan con encogimiento (TELIS-ROMERO; SOBRAL, 2003).

Aun cuando se tengan en cuenta modelos macroscópicos simplificados donde se pueden despreciar las resistencias internas, el flujo de agua se puede expresar con ecuaciones del tipo (Ecuación 1) (KEEY, 1978):

$$
F_{a}=\frac{1}{A} d\left(V \rho_{s} X\right) / d t
$$

donde $F_{\mathrm{a}}$ es el flujo de agua, $A$ y $V$ el área y el volumen de la muestra, respectivamente, $\rho_{\mathrm{s}}$ la densidad del sólido y $X$ el contenido de humedad de la muestra. Para un lecho (Ecuación 2):

$V=(1-E) V_{1}$

donde $E$ es la porosidad del lecho y $V_{1}$ su volumen. Estas propiedades pueden cambiar a medida que las partículas que componen el lecho se deshidratan.

Según la bibliografía relevada (CRAPISTE; WHITAKER; ROTSTEIN, 1988; KILPATRICK; LOWE; VAN ARSDEL, 1975; LAZAR; FARKAS, 1971; LOZANO; ROTSTEIN; URBICAIN, 1983; OCHOA et al., 2002a,b; PEZZUTTI, 1994; RATTI, 1991;1994; SUZUKI et al., 1976; VULLIOUD; MÁRQUEZ; DE MICHELIS, 1999), aún no se ha logrado generalizar un modelo que permita evaluar los cambios, probablemente debido a que el encogimiento depende no sólo de la composición, sino también de la estructura y de las fuerzas mecánicas que se desarrollan durante la ocurrencia del mismo. Algunos autores (SHISHIDO; SUZUKI; OHTANI, 1986; TOWNER, 1987), estudiaron el encogimiento partiendo de las leyes de la mecánica y analizando las tensiones y deformaciones a las que está sometido el material, pero referido a secado de suelos, arcillas y polímeros. Para productos biológicos, éste es un problema extremadamente complejo, debido a la naturaleza multifásica del sistema celular, y a que se requiere el conocimiento de las propiedades estructurales, mecánicas y elásticas de los distintos componentes del mismo y su variación con el contenido de agua y la temperatura (RATTI, 1991).

Por ello, todavía es necesario evaluar los cambios experimentalmente y luego con ellos encontrar modelos para su conveniente descripción matemática. El objetivo de éste trabajo es entonces la obtención experimental de los cambios de volumen de lecho para frutos del cerezo (Prunus avium), del guindo (Prunus cerasus) y de la rosa mosqueta (Rosa eglanteria), y, mediante la utilización de modelos previamente desarrollados por Ochoa et al. (2002a,b) para el encogimiento volumétrico de las partículas individuales, proponer ecuaciones para evaluar el encogimiento de volumen y la porosidad de los lechos estudiados.

\section{Material y métodos}

\subsection{Materia prima}

Se utilizaron frutos frescos de cerezo, var. Napolitana ( ${ }^{\circ}$ Brix entre 19 y 21 ; humedad en base seca entre 3,54 y 3,31 ), de guindo, var. Montmorency $\left({ }^{\circ}\right.$ Brix entre 18,5 y 20 ; humedad en base seca entre 3,39 y 3,14 ) y de rosa mosqueta $\left({ }^{\circ}\right.$ Brix entre 15,4 y 17; humedad en base seca entre 1,01 y 0,93 ) de recolección silvestre. Los mismos se almacenaron en una cámara frigorífica con aire forzado de alta humedad, a una temperatura de $4{ }^{\circ} \mathrm{C}$, hasta su utilización.

\subsection{Determinación del contenido de humedad de las muestras}

El contenido inicial se determinó seleccionando 50 frutos tomados al azar, con dos repeticiones, para cada fruta y se colocaron en estufa de vacío a $60{ }^{\circ} \mathrm{C}$ hasta peso constante. El contenido de humedad de las muestras parcialmente deshidratadas se obtenía por diferencia de peso. Las muestras se pesaban con una balanza analítica $( \pm 0,001 \mathrm{~g})$.

\subsection{Experiencias de deshidratación de lechos de partículas}

Para las experiencias se utilizó un equipo de secado experimental previamente desarrollado (OCHOA et al., 2002a,b), que permitía regular las variables operativas de secado, cuyo esquema se presenta en la Figura 1. La cámara de secado, de sección circular, permitía alturas de lecho de hasta $0,12 \mathrm{~m}$, y las experiencias se llevaron a cabo con las siguientes condiciones operativas: temperatura del aire de secado 50,60 y $70{ }^{\circ} \mathrm{C}$; velocidad del aire: 1,2 y $4 \mathrm{~m} / \mathrm{s}$; humedad relativa del aire: 5 y $50 \%$; y la altura del lecho entre 0,04 y $0,12 \mathrm{~m}$. En el tiempo inicial y durante la deshidratación se medía, a intervalos regulares, la altura del lecho con 8 escalas graduadas colocadas simétricamente en la cámara de secado, siendo la altura tomada promedio de las ocho escalas, y el peso de las muestras correspondientes. Con los valores obtenidos se calculaba el volumen de lecho. Mediante la estimación del volumen de partículas en el lecho, con modelos previamente desarrollados, y los valores del volumen obtenidos

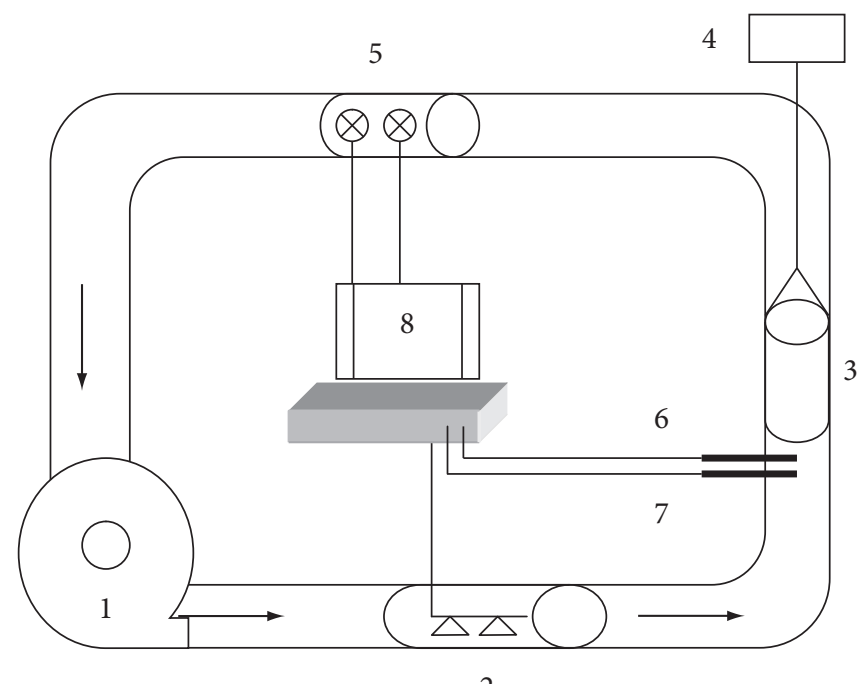

2

Figura 1. Esquema del equipo experimental de secado. 1: Ventilador; 2: estación de humectación del aire; 3: cámara de secado; 4: balanza; 5: calefactor eléctrico del aire; 6 : sensor de temperatura de bulbo seco del aire; 7: sensor de humedad relativa del aire; y 8: controlador automático de la temperatura del aire de secado. 
experimentalmente, se estimaba la porosidad. Asimismo, y mediante regresión de los valores experimentales, se obtuvieron modelos para predecir el volumen y la porosidad de los lechos.

\section{Resultados y discusión}

\subsection{Cambios en el volumen de lecho}

En la Figura 2 se presentan los resultados experimentales obtenidos para los cambios de volumen de lecho de cereza y guinda, en función del contenido de humedad, y la predicción de la Ecuación 3. Los resultados para estas dos frutas se presentan en el mismo gráfico, ya que el análisis estadístico (ANOVA, 1\%) efectuado con el software Origin v. 4.1, indica que no existen diferencias significativas entre ellos, como se indica en la Tabla 1, junto con las medias y la varianza de varios sets de ensayos. Los datos obtenidos se regresionaron con un polino-

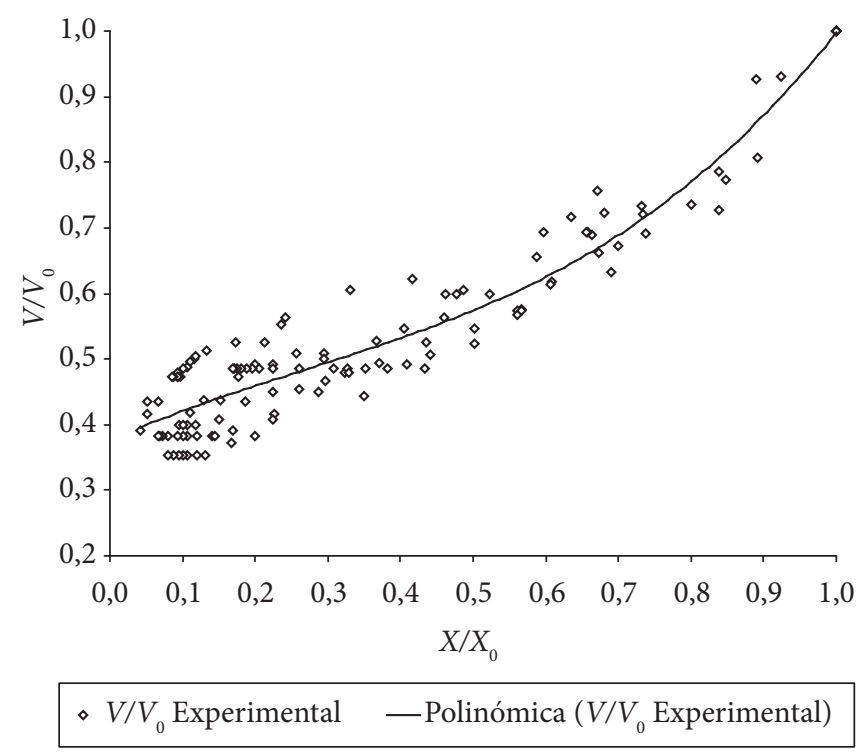

Figura 2. Cambios del volumen adimensional experimental de lecho de cereza y guinda en función del contenido de humedad adimensional de las muestras y la correspondiente polinómica de regresión Ecuación 3. mio de orden 3, atendiendo a las recomendaciones efectuadas por Ratti $(1991,1994)$, ya que la recomendación efectuada por Telis-Romero y Sobral (2003) no satisfacía convenientemente usando los datos experimentales de este trabajo. La ecuación obtenida para el cambio de volumen de lecho en cerezas y guindas se presenta en la Ecuación 3:

$\frac{V_{l}}{V_{l 0}}=0,7598\left(\frac{X}{X_{0}}\right)^{3}-0,6698\left(\frac{X}{X_{0}}\right)^{2}+0,5299\left(\frac{X}{X_{0}}\right)+0,3795$

con $R^{2}=0,93$ y Error \% comprendido entre $-16,4$ y 23,3 , siendo $X / X_{0}$ la relación entre la humedad actual y la inicial de las muestras y $V_{1} / V_{\text {lo }}$ la relación entre el volumen de lecho actual y el inicial.

En la Figura 3 se muestra el cambio de volumen de lecho para frutos de la rosa mosqueta y la predicción de la Ecuación 4. Los datos obtenidos se regresionaron con un polinomio de orden 3, atendiendo a las mismas recomendaciones efectuadas por Ratti $(1991,1994)$ y porque se obtenía un mejor coeficiente de

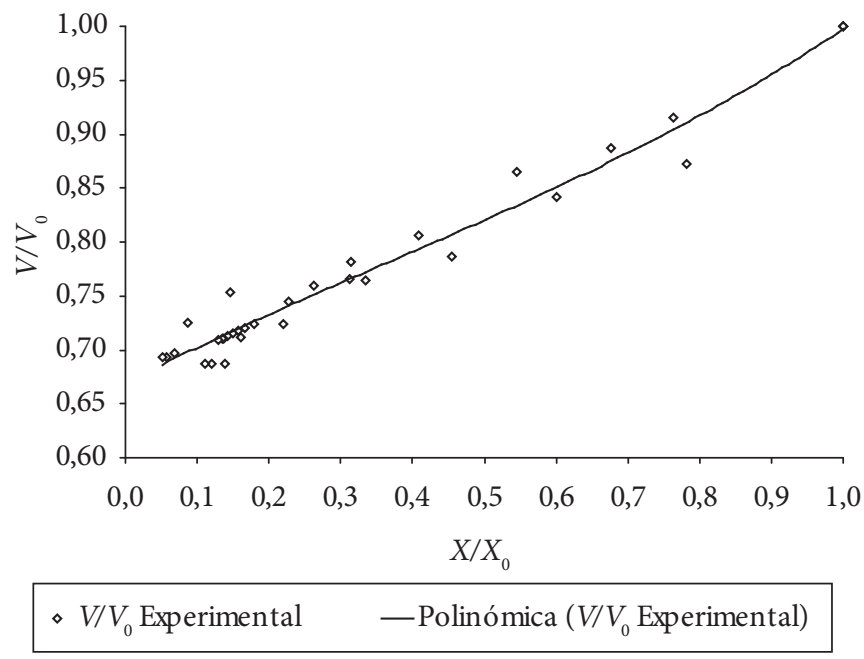

Figura 3. Cambios del volumen adimensional de lecho de los frutos de la rosa mosqueta en función del contenido de humedad adimensional de las muestras y la correspondiente polinómica de regresión Ecuación 4.

Tabla 1. Análisis estadístico de los cambios de volumen y porosidad de lecho para cerezas y guindas.

\begin{tabular}{|c|c|c|c|c|c|c|c|}
\hline \multicolumn{4}{|c|}{ ANOVA sobre $V / V o$} & \multicolumn{4}{|c|}{ ANOVA sobre $E / E o$} \\
\hline Dato & Media & Varianza & $\mathrm{N}$ & Dato & Media & Varianza & $\mathrm{N}$ \\
\hline$V / V_{0}$ Cereza & 0,53102 & 0,04232 & 10 & $E / E_{0}$ Cereza & 1,07378 & 0,03412 & 10 \\
\hline$V / V_{0}$ Cereza & 0,50007 & 0,04799 & 16 & $E / E_{0}$ Cereza & 1,09710 & 0,01966 & 16 \\
\hline$V / V_{0}$ Guinda & 0,62464 & 0,04126 & 7 & $E / E_{0}$ Guinda & 1,27189 & 0,0503 & 7 \\
\hline$V / V_{0}$ Guinda & 0,57382 & 0,03356 & 13 & $E / E_{0}$ Guinda & 1,27521 & 0,08471 & 7 \\
\hline$V / V_{0}$ Cereza & 0,58333 & 0,02086 & 15 & $E / E_{0}$ Cereza & 1,16772 & 0,00631 & 15 \\
\hline$V / V_{0}$ Cereza & 0,55284 & 0,02348 & 16 & $E / E_{0}$ Cereza & 1,13838 & 0,0369 & 16 \\
\hline$V / V_{0}$ Cereza & 0,56382 & 0,01883 & 22 & $E / E_{0}$ Cereza & 1,06537 & 0,06524 & 22 \\
\hline$V / V_{0}$ Guinda & 0,50013 & 0,03262 & 14 & $E / E_{0}$ Guinda & 1,11602 & 0,0562 & 14 \\
\hline$V / V_{0}$ Cereza & 0,61111 & 0,02673 & 12 & $E / E_{0}$ Cereza & 0,95333 & 0,01451 & 12 \\
\hline \multicolumn{4}{|c|}{$F=0,7697 p=0,63008$} & \multicolumn{4}{|c|}{$\mathrm{F}=2,52238 \mathrm{p}=0,01474$} \\
\hline \multicolumn{4}{|c|}{ Al nivel 0,01 , las medias no son significativamente diferentes. } & \multicolumn{4}{|c|}{ Al nivel 0,01 , las medias no son significativamente diferentes. } \\
\hline
\end{tabular}


regresión y menores errores absolutos que con una lineal como la recomendada por Telis-Romero y Sobral (2003). La ecuación obtenida para el cambio de volumen de lecho en frutos de la rosa mosqueta se presenta en la Ecuación 4:

$$
\frac{V_{l}}{V_{l 0}}=0,728\left(\frac{X}{X_{0}}\right)^{3}-0,1392\left(\frac{X}{X_{0}}\right)^{2}+0,3391\left(\frac{X}{X_{0}}\right)+0,6694
$$

con $\mathrm{R}^{2}=0,97$ y Error \% comprendido entre - 4,9 y 4,4.

Como se puede observar en las Figuras 2 y 3, el volumen adimensional del lecho sólo es función del contenido de agua de la muestra, y no poseen influencia estadísticamente significativas sobre sus valores la temperatura, velocidad, humedad relativa del aire de secado y la altura de lecho.

\subsection{Cálculo de la porosidad de lecho para cereza, guinda y rosa mosqueta}

La porosidad de lecho se define como se indica en la Ecuación 5:

$$
E=1-\frac{N V_{p}}{V_{l}} \quad ; \quad E_{0}=1-\frac{N V_{p 0}}{V_{l 0}}
$$

donde $E$ y $E_{0}$ corresponden a la porosidad del lecho parcialmente deshidratado y la inicial, respectivamente; $V_{\mathrm{p}}$ y $V_{\mathrm{p} 0}$ corresponden al volumen de la partícula individual parcialmente deshidratada e inicial, respectivamente, y $N$ al número total de partículas en el lecho. $V_{1}$ y $V_{10}$ son el volumen de lecho parcialmente deshidratado e inicial, respectivamente. El volumen de la partícula individual, que cambia a medida que el producto se deshidrata, debido a los fenómenos de encogimiento, se obtuvo de correlaciones anteriormente desarrolladas por Bernardi et al. (2001a,b) y Ochoa (2002a,b), y el volumen de lecho, de las Ecuaciones 3 y 4. Los valores experimentales obtenidos se presentan en la Figura 4, para guindas y cerezas, y en la Figura 5, para frutos de la rosa mosqueta, junto con la predicción de la Ecuación 5, en función de la relación entre la humedad de las partículas parcialmente deshidratadas y la humedad inicial de las mismas $\left(X / X_{0}\right)$. Del mismo modo que para el volumen de lecho, el análisis estadístico indica que la porosidad de lecho para guindas y cerezas no presenta diferencias significativas, como se muestra en la Tabla 1. Tampoco se encontraron, como es lógico, diferencias significativas entre tratamientos, ya que tanto los cambios de volumen de lecho como los de volumen de las partículas individuales no dependían de las variables operativas del secado (BERNARDI et al., 2001a,b; OCHOA, 2002a,b).

Los errores porcentuales obtenidos entre los valores experimentales y la predicción de la Ecuación 5 fueron, para cereza y guinda, entre $-15,2$ y 21,$1 ; y$, para rosa mosqueta, entre $-2,6$ y 6,1 .

Como se puede ver en la Figura 4, la porosidad de lecho para cerezas y guindas disminuye hasta alcanzar aproximadamente un valor de $X / X_{0}$ igual a 0,7 , y luego comienza a aumentar.

Según datos reportados en la bibliografía para otras frutas y hortalizas (RATTI, 1991,1994; TELIS-ROMERO; SOBRAL, 2003), la porosidad de lecho aumenta a medida que disminuye el contenido de humedad, como consecuencia de que el encogi- miento de las partículas individuales solamente es uniforme en las primeras etapas del secado, $\mathrm{y}$ a tiempos mayores se producen rugosidades, plegamientos y deformaciones poco uniformes, lo que implica un encogimiento diferencial que provoca el aumento de la porosidad.

Esta conclusión fue extraída de datos experimentales de partículas de geometría no esférica (rodajas, cilindros y cubos)

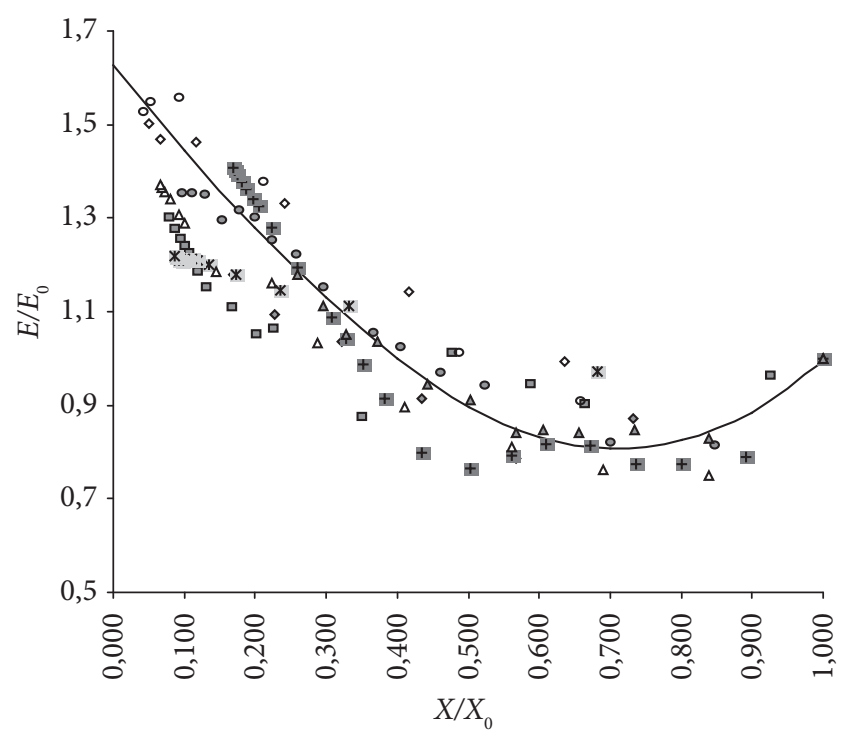

\begin{tabular}{|ccc|}
\hline - $E / E_{0}$ Cereza & $\square E / E_{0}$ Cereza & $\diamond E / E_{0}$ Guinda \\
$\bullet E / E_{0}$ Cereza & $\neq E / E_{0}$ Cereza & $\Delta E / E_{0}$ Guinda \\
$\circ E / E_{0}$ Guinda & $* E / E_{0}$ Cereza \\
$\Delta E / E_{0}$ Cereza & $-E / E_{0}$ Estimada \\
\hline
\end{tabular}

Figura 4. Porosidad de lecho adimensional de guindas y cerezas en función del contenido de humedad adimensional de los frutos y la predicción de la Ecuación 5.

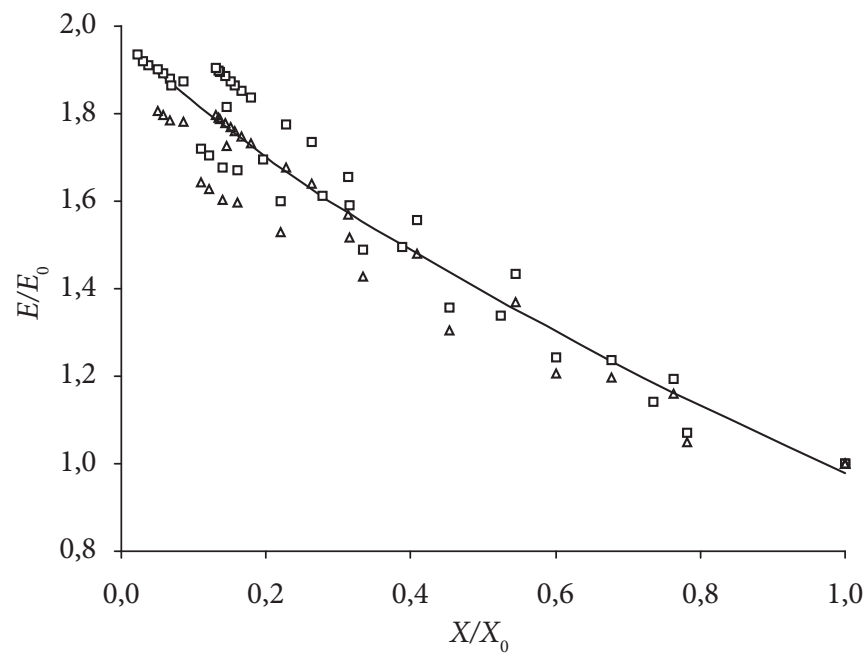

Figura 5. Porosidad de lecho adimensional en función del contenido de humedad adimensional para frutos de la rosa mosqueta y la predicción de la Ecuación 5. 
(RATTI, 1991,1994; TELIS-ROMERO; SOBRAL, 2003). Para partículas muy similares a una esfera, como las cerezas y guindas (esfericidad de las cerezas frescas entre 0,92 y 0,96 ; esfericidad de las guindas frescas entre 0,94 y 0,97), el fenómeno ocurre de un modo similar, pero probablemente debido a la adherencia entre partículas, ya que el fruto, excepto en la etapa de disminución de la porosidad, permanece con formas muy próximas a la esfera y la porosidad en lechos de esferas es prácticamente independiente del tamaño. Observaciones cualitativas muestran que las frutas presentan un encogimiento muy uniforme en las primeras etapas del secado, sin signos de rugosidades, plegamientos, etc. Por ello, la porosidad debería cambiar muy poco, ya que la porosidad de lechos homogéneos, sin efectos de borde, de partículas casi esféricas, es prácticamente constante. Sin embargo, también se observa en las primeras etapas del secado que las cerezas y guindas se ablandan significativamente por el efecto de la temperatura, hasta un contenido de humedad adimensional de aproximadamente 0,7 . Como consecuencia de que la velocidad de secado es relativamente baja (entre 3 y 4,5 horas para llegar al valor de humedad indicado), ocurre un colapso momentáneo del lecho con cambios relativamente pequeños del contenido de humedad disminuyendo la porosidad. Este fenómeno se produce por el cambio de forma de las partículas individuales (pierden la forma cuasi esférica por aplastamiento como consecuencia del ablandamiento térmico y, justamente en ese rango, disminuye significativamente el denominado factor de forma de Heywood, citado por Panyawong y Devahastin (2007). En consecuencia, la velocidad de disminución del volumen del lecho es mayor que la velocidad de cambio del volumen de las partículas individuales. Luego, para contenidos de humedad adimensional menores que 0,7 , las frutas se vuelven más rígidas, recuperando su forma casi esférica, continuando su encogimiento de forma normal, por lo que vuelve a aumentar la porosidad. Este efecto explicaría la disminución de porosidad observada en guindas y cerezas hasta un contenido de humedad de aproximadamente 0,7 . Dicho comportamiento requiere especial atención, ya que también afecta aspectos del diseño mecánico de equipos, como la potencia de soplado del aire de secado, la cual depende de la pérdida de carga y esta a su vez de la porosidad (TELIS-ROMERO; SOBRAL, 2003). Si la porosidad disminuye, aumenta proporcionalmente la potencia necesaria de soplado.

Mientras que, el comportamiento de la porosidad de los frutos de la rosa mosqueta, de forma ovoide, con esfericidad para el fruto fresco entre 0,54 y 0,58 , concuerda con los datos bibliográficos relevados para otros productos.

Los cambios de porosidad y volumen de lecho sólo son influidos por el contenido de humedad de las partículas y probablemente el efecto de las variables operativas del secado se ve enmascarado dentro de los errores experimentales. Los resultados fueron regresionados con modelos basados en información bibliográfica, con concordancia que se considera satisfactoria. Si bien los errores porcentuales en el caso de los cambios de volumen y porosidad de los lechos de cerezas y guindas se encuentran entre $-16,4$ y 23,3 , y pueden considerarse elevados, en realidad el apartamiento indicado se encuentra para valores de contenido de humedad $X / X_{0}$ menores que 0,2 , lo que equivale a una humedad en base seca menor que 0,18.
De acuerdo a las curvas de equilibrio sorcional para las mismas frutas (VULLIOUD; MÁRQUEZ; DE MICHELIS, 2004), para una actividad acuosa de 0,6 , la humedad de equilibrio en base seca es de 0,39 a $20^{\circ} \mathrm{C}$, por ello estas frutas nunca se secan hasta los tenores de humedad indicados. Para valores de $X / X_{0}$ superiores a 0,2 , los errores porcentuales se encuentran comprendidos entre $-9,8$ y 10,7 . La porosidad de lechos de cerezas y guindas presenta un comportamiento atípico, atribuido a la deformación por ablandamiento térmico de dichas frutas y a la relativamente baja velocidad de secado en las primeras etapas de la deshidratación; en este caso, por el comportamiento observado, la pérdida de carga se incrementa alrededor de tres veces, según datos publicados para otros productos (TELIS-ROMERO; SOBRAL, 2003), por ende la potencia de soplado debería incrementarse proporcionalmente. Asimismo, aún no es posible predecir parámetros importantes para el diseño, como la pérdida de carga a través de los lechos, sin el conocimiento de la porosidad de los mismos. La porosidad de lechos de los frutos de la rosa mosqueta, de forma ovoide, sigue un comportamiento similar al reportado por otros autores para otros tipos de alimento y geometrías de partícula poco esféricas.

\section{Conclusiones}

Se determinó experimentalmente el cambio de volumen y la porosidad para lechos de cerezas, guindas y frutos de la rosa mosqueta y se modelaron los cambios con ecuaciones muy simples.

Las cerezas y guindas presentan cambios de volumen y de porosidad que pueden incluirse dentro del mismo modelo matemático, ya que estadísticamente no difieren entre sí.

Las ecuaciones presentadas pueden ser utilizadas en la simulación y/u optimización de secadoras industriales.

\section{Agradecimientos}

Los autores agradecen a la Universidad Nacional del Comahue, Facultad de Ingeniería, Asentamiento Universitario Villa Regina (Proyecto FAIN I125), a la Agencia Nacional de Ciencia y Tecnología (Proyecto PICT 09-06427) y al CONICET (Proyecto PIP 5511) por el financiamiento de este trabajo; a la AER El Bolsón del INTA y a CORFO - CHUBUT por la colaboración prestada en la recolección de muestras, infraestructura, etc.

El autor A. De Michelis es miembro del CONICET.

\section{Referencias}

BERNARDI, I. et al. Changes of volume and area during air dehydration of whole rose hip fruits. In: PROCEEDING OF ENPROMER, 16 al 20 de septiembre de 2001, v. III, Santa Fé, Argentina. p. 1447-1452. Anales... Santa Fé, Argentina: Centro Regional de Investigación y Desarrollo de Santa Fe (Ceride), 2001.

. Cambios de volumen y área durante la deshidratación de frutos enteros del prunus cerasus. In: MEMORIAS DEL $8^{\circ}$ CONGRESSO LATINOAMERICANO DE TRANSFERÊNCIA DE CALOR Y MATERIA, 2001b, Veracruz, México. p. 197-200. Anales... Veracruz: Instituto Tecnológico de Veracruz, 2001 b. 
CRAPISTE, G. H.; WHITAKER, S.; ROTSTEIN, E. Drying of cellular material. Chemical Engineering Science, London, v. 43, n. 11, p. 2919-2928, 1988.

KEEY, R. B. Introduction to industrial drying operations. London: Pergamon Press, 1978.

KILPATRICK, P. W.; LOWE, E.; VAN ARSDEL, W. B. Tunnel dehydration for fruit and vegetables. Advances in Food Research, New York and London, v. 50, p. 313-372, 1975.

LAZAR, M. E.; FARKAS, D. F. The centrifugal fluidized bed. 2. Drying studies on pieces-form foods. Journal of Food Science, New York and London, v. 36, n. 2, p. 315-320, 1971.

LOZANO, J. E.; ROTSTEIN, E.; URBICAIN, M. J. Shrinkage, porosity and bulk density of foodstuffs at changing moisture content. Journal of Food Science, New York and London, v. 48, n. 5, p. 1497-1502, 1553, 1983.

Total porosity and open-porosity in the drying of fruits. Journal of Food Science, New York and London, v. 45, n. 5, p. 1403-1407, 1980.

OCHOA, M. R. et al. Volume and area shrinkage during dehydration of whole sour cherry fruits (prunus cerasus). Drying Technology, New York, n. 20, v. 1, p. 147-156, 2002a.

Shrinkage during convective air drying of whole rose hip (Rosa Rubiginosa L.) fruits. Lebensmittel-Wissenschaft und-Technologie, Oxford, v. 35, n. 5, p. 400-406, 2002 b.

PANYAWONG, S.; DEVAHASTIN, S. Determination of deformation of a food product undergoing different drying methods and conditions via evolution of a shape factor. Journal of Food Engineering, Essex, v. 78, n. 1, p. 151-161, 2007.
PEZZUTTI, A. Deshidratación de ajo y cebolla. Argentina, 1994. p.212. Tese - (Doutorado em Ingeniería Química). Universidad Nacional del Sur.

RATTI, C. Diseño de secaderos de productos frutihorticolas. Argentina, 1991.p.205. Tese - (Doutorado em Ingeniería Química). Universidad Nacional del Sur.

Shrinkage during drying of foodstuffs. Journal of Food Engineering, Essex, v. 23, n. 1, p. 91-105, 1994.

SHISHIDO, I.; SUZUKI, M.; OHTANI, S. On the drying mechanism of shrinkage materials. In: WORLD CONGRESS III OF CHEMICAL ENGINEERING, 1986, Tokyo. Paper... Tokyo, 1986, September. p. 21-25.

SUZUKI, K. et al. Shrinkage in dehydration of root vegetables. Journal of Food Science, New York and London, v. 41, n. 5, p. 1189-1193, 1976.

TELIS-ROMERO, J.; SOBRAL, P. J. do A. Caída de presión en lecho fijo de cubos de papa, remolacha y zanahoria considerando encogimiento. Ciencia e Tecnología de Alimentos, São Paulo, v. 23, Supl., p. 110-115, Dez. 2003.

TOWNER, G. D. The tensile stress generated in clay through drying. Journal of Agricultural and Engineering Research, New York and London, v. 37, n. 3-4, p. 279-285, 1987.

VULLIOUD, M.; MÁRQUEZ, C. A.; DE MICHELIS, A. Deshidratación y cambios de volumen en frutos no tradicionales. In: VIII CONGRESSO ARGENTINO DE CIÊNCIA Y TECNOLOGÍA DE ALIMENTOS, 1999, Rafaela, Santa Fe. Actas... Rafaela, Santa Fe, 1999. CD-ROM N ${ }^{\circ} 01-04$.

. Desorption isotherms for sweet and sour cherry. Journal of Food Engineering, Essex, v. 63, n. 1, p. 15-19, 2004. 